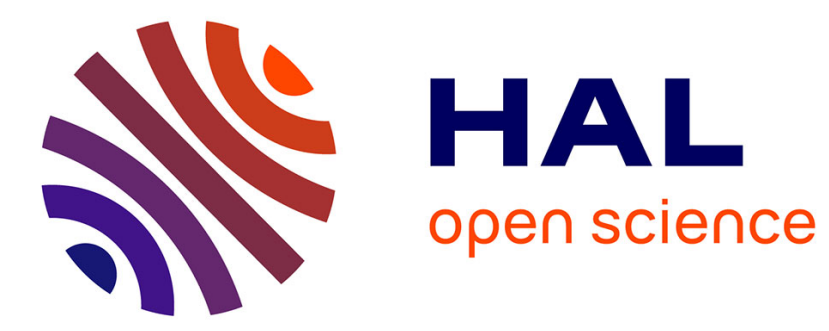

\title{
Decentralized synchronization of time-varying oscillators under time-varying bidirectional graphs
}

Mohamed Maghenem, Herman Lekefouet, Antonio Loria, Elena Panteley

\section{To cite this version:}

Mohamed Maghenem, Herman Lekefouet, Antonio Loria, Elena Panteley. Decentralized synchronization of time-varying oscillators under time-varying bidirectional graphs. ACC 2019 - American Control Conference, Jul 2019, Philadelphie, PA, United States. pp.4018-4023, 10.23919/ACC.2019.8815146 . hal-02367890

\section{HAL Id: hal-02367890 \\ https://hal.science/hal-02367890}

Submitted on 5 Mar 2020

HAL is a multi-disciplinary open access archive for the deposit and dissemination of scientific research documents, whether they are published or not. The documents may come from teaching and research institutions in France or abroad, or from public or private research centers.
L'archive ouverte pluridisciplinaire HAL, est destinée au dépôt et à la diffusion de documents scientifiques de niveau recherche, publiés ou non, émanant des établissements d'enseignement et de recherche français ou étrangers, des laboratoires publics ou privés. 


\title{
Decentralized synchronization of time-varying oscillators under time-varying bidirectional graphs
}

\author{
Mohamed Maghenem \\ Hermann Lekefouet \\ Antonio Loría \\ Elena Panteley
}

\begin{abstract}
We study the synchronization problem for a network of planar harmonic oscillators with time-varying frequency. The oscillators are interconnected using a timevarying bidirectional graph. That is, the interconnections may be interrupted over some intervals of time, but a certain persistent connectivity prevails. We provide tight sufficient conditions on the graph's connectivity to guarantee uniform exponential synchronization of the network. Our main results are based on original statements of stability for linear timevarying systems under persistency-of-excitation conditions and Lyapunov's direct method.
\end{abstract}

\section{INTRODUCTION}

Decentralized coordination control for networked systems consists in designing a control law for each system in order to achieve a coordinated task or, more concretely, to make certain quantities of interest in each system reach a common value [1]. For instance, it may be required for these systems to reach a certain geometric pattern [2], [3], follow a target or a leader [4], [5], or also describe a common steady-state behavior [6], [7], [8]. When the control law is decentralized, the input of each system is designed using only local information provided by a set of agents called neighbors. That is, the overall interconnectivity of the network is characterized by a graph [9].

In this context, an interesting challenge arises when the communication is constrained. For instance, when the communication is intermitent or when the graph topology is time-varying, or even when both happen simultaneously [10]. Such a problems have received a considerable attention in the literature starting with [6], [11], where a network of single-integrator systems is considered and the interconnection graph is assumed to be piece-wise constant and to switch between a finite number of undirected and directed graphs, respectively. Extensions of these works to the case of harmonic oscillators with a constant frequency are presented in [12] and [13] under directed and undirected graphs, respectively. See also [14], [15] and [16], [17], where a network of single integrators under generic time-varying directed and bidirectional graphs are considered, respectively. Furthermore, in [18] and among many other references, networks of linear time-invariant systems are considered under general time varying communication graphs.

This article is supported by Government of Russian Federation (grant 074-U01). M. Maghenem is with the University of California at Santa Cruz, USA. Email: mmaghene@ucsc.edu. H. Lekefouet is with Alinda Technologies, France. Email: hermann.lekefouet@gmail.com. A. Loría and E. Panteley are with the CNRS. LSS-CentraleSupelec, 3 Rue Joliot Curie, 91192, France. Email: loria,panteley@1ss.supelec.fr. E. Panteley is also with ITMO University, Kronverkskiy av. 49, Saint Petersburg, 197101, Russia.
In this paper, we study the decentralized synchronization problem for a network planar identical harmonic oscillators, the oscillators are assumed to have an identical but timevarying frequency. and they are interconnected according to a general time-varying bidirectional graph. We assume that the communication is not perennial, but it may be interrupted over intervals of time of minimal length (persistent connectivity). More precisely, we suppose that there exists a spanning tree in the graph topology whose edge weights verify a certain form of persistency of excitation relatively to the natural frequency of the oscillators. To the best of our knowledge, this is the first result on synchronization of linear time-varying systems using a general time-varying graph. The sufficient conditions to guarantee uniform exponential synchronization with an explicit estimate of the convergence rate are interpreted in terms of graph connectivity. The relevance of our main results to control applications is illustrated by a simple network of nonholonomic systems. From a technical viewpoint, our analysis method relies on stability statements for gradient systems in the context of consensus - see [16]; it consists in finding adequate error coordinates such that uniform global exponential stability (UGES) in those coordinates is equivalent to uniform synchronization in the original coordinates. Interestingly, as we will show, the resulting linear time-varying system in the error coordinates has a particular structure that extends the class of systems typically encountred in the literature of model reference adaptive control [19], [20], [21]. Therefore, from a technical viewpoint, our main statements may serve as basics to address the general case of nonlinear networks where the oscillators' frequency is replaced by a general nonlinear function.

The core of the paper lies in the next section, where we state and motivate the synchronization problem, we present some technical well-known facts about graph theory, and our main results. In Section III, we present illustrative simulation results and we conclude with some remarks in Section IV.

Due to space limitations, some proofs are omitted and will be published elsewhere.

\section{SYNCHRONIZATION OF HARMONIC OSCILLATORS}

\section{A. Problem statement and motivation}

Let us consider a set of $n$ identical controlled planar harmonic oscillators with time-varying frequency of the form

$$
\left[\begin{array}{c}
\dot{x}_{i} \\
\dot{z}_{i}
\end{array}\right]=\left[\begin{array}{cc}
0 & -b(t) \\
b(t) & 0
\end{array}\right]\left[\begin{array}{c}
x_{i} \\
z_{i}
\end{array}\right]+\left[\begin{array}{l}
1 \\
0
\end{array}\right] u_{i} i \in\{1, \ldots, n\} .
$$


The frequency of oscillation, denoted above by $b$ is assumed to be piece-wise continuous with respect to time. It is also assumed that the oscillators are interconnected according to a bidirectional time-varying graph. More precisely, the control input for each node is given by

$$
u_{i}(t):=\sum_{j=1}^{n} a_{i j}(t)\left(x_{j}-x_{i}\right),
$$

where $a_{i j}(t) \geq 0$ corresponds to the coupling between the $i$-th and the $j$-th nodes. Hence, it is assumed that the interconnections are time-varying and may be lost over intervals of time, but they verify a certain form of persistency of excitation [19].

The problem of interest is to establish sufficient conditions in order to synchronize the considered network uniformly and exponentially according to the following definition.

Definition 1: The network of time-varying systems in (1) is said to be uniformly exponentially synchronized if there exists a linear change of coordinates $e:=T\left[\begin{array}{ll}x & z\end{array}\right]^{\top} \in \mathbb{R}^{n-1}$, with $x:=\left[x_{1} \cdots x_{n}\right]$ and $z:=\left[z_{1} \cdots z_{n}\right]$, such that

$$
\left[\begin{array}{ll}
x_{1} & z_{1}
\end{array}\right]=\left[\begin{array}{ll}
x_{2} & z_{2}
\end{array}\right]=\ldots=\left[\begin{array}{ll}
x_{n} & z_{n}
\end{array}\right] \Longleftrightarrow e=0,
$$

and the origin $\{e=0\}$ is uniformly globally exponentially stable.

To the best of our knowledge, this problem is unsolved and yet, it has clear motivations in the field of control of cooperative systems, as we illustrate through the following concrete example of a network of nonholonomic systems required to perform a synchronized motion on the plane.

Example 1: Consider a group of $n$ mobile robots modeled by the kinematics equations

$$
\begin{aligned}
& \dot{x}_{i}=u_{i} \cos (\theta)_{i} \\
& \dot{y}_{i}=u_{i} \sin (\theta)_{i} \\
& \dot{\theta}_{i}=\quad b_{i} \quad i \in\{1, \ldots, n\},
\end{aligned}
$$

where $u_{i}$ and $b_{i}$ are the forward and angular velocities, respectively. The first two elements of $\left[\begin{array}{ll}x_{i} & y_{i} \\ \theta_{i}\end{array}\right]^{\top}$ correspond to the Cartesian coordinates of a point on the robot with respect to a fixed reference frame, and $\theta_{i}$ denotes the robot's orientation with respect to the same frame. Consider the problem of position synchronization for the network in (3). We assume, without loss of generality, that the robots initially have the same orientation and that $b_{1}=b_{2}=\ldots=b_{n}:=b^{1}$. To this end, we first transform the coordinates of each robot from the global to a local coordinate frame related to each robot, that is, we define

$$
\left[\begin{array}{l}
x_{l i} \\
y_{l i}
\end{array}\right]:=\left[\begin{array}{cc}
\cos \theta_{i} & \sin \theta_{i} \\
-\sin \theta_{i} & \cos \theta_{i}
\end{array}\right]\left[\begin{array}{l}
x_{i} \\
y_{i}
\end{array}\right] .
$$

In these new coordinates, the kinematics equations become

$$
\begin{aligned}
\dot{\theta}_{i} & =b(t) \\
\dot{x}_{l i} & =b(t) y_{l i}-u_{i} \\
\dot{y}_{l i} & =-b(t) x_{l i} ;
\end{aligned}
$$

\footnotetext{
${ }^{1}$ Otherwise, a decentralized feedback control can be used to synchronize the orientations independently of the positions.
}

note that the transnational equations (5b)-(5c) correspond exactly to the equations (1).

\section{B. Preliminaries on algebraic graph theory}

Before stating our main result, some general concepts and notations on algebraic graph theory for time-varying graphs need to be recalled for the sake of clarity and selfcontainedness. The material in this subsection is written after [16], but a more detailed and extensive treatment is given, e.g. in [9].

An undirected graph $\mathcal{G}$ is a pair $(\mathcal{V}, \mathcal{E})$, where $\mathcal{V}=$ $\left\{v_{1}, v_{2}, \cdots, v_{n}\right\}$ is a finite non-empty set of nodes (agents) and $\mathcal{E} \subseteq \mathcal{V} \times \mathcal{V}$ is a set of ordered pairs of nodes forming edges (communication links) denoted $\left\{e_{1}, e_{2}, \cdots, e_{m}\right\}, m \geq$ $n-1$. The pair $\left\{v_{i}, v_{j}\right\} \in \mathcal{E}$ denotes an undirected edge if the agents $v_{i}$ and $v_{j}$ communicate with each other both ways, that is, there exists $k \leq m$ such that $e_{k}$ denotes the corresponding undirected edge. A path in an undirected graph is a finite or infinite sequence of edges that connects a sequence of vertices denoted by $\left\{v_{i_{0}}, v_{i_{1}}, \cdots, v_{i_{p}}\right\}$, where $p \leq n$, which are distinct from each other. A simple cycle is defined as a closed path. A bidirectional graph is said to be connected if for every pair of vertices in $V(\mathcal{G})$ there is a path relating them. A tree is defined as a connected subgraph without any cycle. A spanning tree for a connected graph $\mathcal{G}$ is a tree containing all the vertices of $\mathcal{G}$. For further development, we also recall that the so-called incidence matrix $D(\mathcal{G}) \in \mathbb{R}^{n \times m}$ of an arbitrarily bidirectional graph $\mathcal{G}$ is defined after an arbitrary orientation of the edges $e_{k}$ as $D(\mathcal{G})=\left[d_{i k}\right]$ where

$$
\begin{aligned}
& {\left[d_{i k}\right]=-1 \text { if, } v_{i} \text { is the tail of } e_{k}} \\
& {\left[d_{i k}\right]=1 \text { if, } v_{i} \text { is the head of } e_{k}} \\
& {\left[d_{i k}\right]=0 \text { otherwise. }}
\end{aligned}
$$

The graph Laplacian matrix $L_{\mathcal{G}}(t) \in \mathbb{R}^{n \times n}$ for an arbitrarily time-varying weighted graph $\mathcal{G}$ is defined as

$$
L_{\mathcal{G}}=D(\mathcal{G}) W(t) D(\mathcal{G})^{\top}
$$

where $W(t) \in \mathbb{R}^{m \times m}$ is a diagonal matrix with the edge weights $w_{k}(t)$, for all $k \in\{1,2, \ldots . m\}$, on the diagonal entry. For any arbitrary orientation of a same undirected graph, Eq. (6) gives the same Laplacian matrix, which is symmetric. Likewise, the edge Laplacian $L_{e}(\mathcal{G}) \in \mathbb{R}^{m \times m}$ matrix (with $\left.W(t)=I_{m}\right)$ is defined as,

$$
L_{e}(\mathcal{G})=D(\mathcal{G})^{\top} D(\mathcal{G}) .
$$

Note that the graph edge Laplacian matrix is positive semidefinite; hence, its eigenvalues may be ordered as

$$
0=\lambda_{1}(\mathcal{G}) \leq \lambda_{2}(\mathcal{G}) \leq \cdots \leq \lambda_{n}(\mathcal{G})
$$

\section{Main result}

Let us consider the $n$ oscillators defined in (1). In view of the inputs, which are defined in (2), the oscillators 
are interconnected forming a network according to a timevarying bidirectional graph defined by the Laplacian $L_{\mathcal{G}}$ whose $i j$-th entry is given by

$$
\left[L_{\mathcal{G}}\right]_{i j}= \begin{cases}-\sum_{k \neq i} a_{i k}(t) & \text { if } j=i \\ a_{i j}(t) & \text { if } j \neq i .\end{cases}
$$

and which satisfies (6).

Now, in a compact form, we see that the dynamical equations corresponding to the network's model are

$$
\left[\begin{array}{c}
\dot{x} \\
\dot{z}
\end{array}\right]=\left[\begin{array}{cc}
-L_{\mathcal{G}}(t) & -b(t) I_{n} \\
b(t) I_{n} & 0
\end{array}\right]\left[\begin{array}{l}
x \\
z
\end{array}\right] .
$$

We give mild, but tight, conditions on the frequency $b(t)$ and the Laplacian matrix $L_{\mathcal{G}}(t)$ such that the closed-loop network in (8) synchronizes. Our first hypothesis is simply that both the interconnections and the frequencies of oscillation are bounded.

Assumption 1 (Boundedness): There exists $\bar{a}>0$ such that ${ }^{2}$

$$
\max \left\{\left|L_{\mathcal{G}}\right|_{\infty},|b|_{\infty}\right\} \leq \bar{a} .
$$

The second standing hypothesis is that the interconnections are persistent relatively to the frequency of oscillations. To state this assumption formally, let us recall the identity (6) and let the weight matrix be partitioned as $W(t):=$ blockdiag $\left\{W_{t}(t), W_{c}(t)\right\}$ where $W_{t}(t) \in \mathbb{R}^{(n-1) \times(n-1)}$ corresponds to the edge weights of a spanning tree and $W_{c}(t) \in$ $\mathbb{R}^{(m-n+1) \times(m-n+1)}$ corresponds to the edge weights of the remaining links (cycles).

Assumption 2 (PE on a Dwell Interval): Given a diagonal matrix function $\Omega: \mathbb{R}_{\geq 0} \rightarrow \mathbb{R}^{(n-1) \times(n-1)}$, there exist reals $T>0, T_{0 i}>0, \underline{W}>0$, and $\mu>0$ such that, for every $t \geq t_{0}$ and $i \leq n-1$, there exists $t_{i}^{*} \in[t, t+T]$, such that each diagonal element of $\Omega(t)$, denoted $\Omega_{i}(t)$, satisfies

$$
\begin{gathered}
\Omega_{i}(s) \geq \underline{W} \quad \forall s \in\left[t_{i}^{*}, t_{i}^{*}+T_{0 i}\right], \\
\left|\int_{t_{i}^{*}}^{t_{i}^{*}+T_{0 i}} \Omega_{i}(s) b(s) d s\right| \geq \mu .
\end{gathered}
$$

Roughly speaking, the condition (10) states that there must be a minimal dwell-time interval, starting at any time, during which the interconnections are active; for instance, the interconnections cannot vanish for increasing amounts of time. Furthermore, the condition imposed by (11) is the one relating the persistency of excitation of the interconnections to the frequency of oscillation. Under Assumptions 1-2, we will be to conclude uniform exponential synchronization for (8) using the following lemma.

Lemma 1: Consider the linear time-varying system

$$
\begin{aligned}
& \dot{x}_{1}=-A(t) x_{1}-b(t) I_{n} x_{2} \\
& \dot{x}_{2}=b(t) I_{n} x_{1},
\end{aligned}
$$

\footnotetext{
${ }^{2}$ For a piece-wise continuous function $\phi: \mathbb{R}_{>0} \rightarrow \mathbb{R}^{n \times m}$, we define $|\phi|_{\infty}:=\sup _{t \geq 0}|\phi(t)|$ where $|\cdot|$ denotes the Euclidean norm of vectors or the induced norm of matrices, depending on the context.
}

where $x_{1}, x_{2} \in \mathbb{R}^{n}, A: \mathbb{R}_{\geq 0} \rightarrow \mathbb{R}^{n \times n}$, and $b: \mathbb{R}_{\geq 0} \rightarrow \mathbb{R}$, and for this system the following statement holds.

The origin for the system (12) is uniformly globally exponentially stable if:

(H1) there exists $\bar{a}>0$ such that

$$
\max \left\{|A|_{\infty},|b|_{\infty}\right\} \leq \bar{a},
$$

(H2) there exist a constant orthogonal matrix $V \in \mathbb{R}^{n \times n}$ and a time-varying diagonal matrix $\Omega(t) \in \mathbb{R}^{n \times n}$ such that

$$
A(t)-V^{\top} \Omega(t) V \geq 0,
$$

and Assumption 2 holds.

Lemma 1 is an original statement of interest in its own right. Indeed, in the following, we provide an example that shows that condition (10) is tight, even in the case of a lowerdimensional system.

Example 2: Consider the system (12) with $n=1$, i.e., let

$$
\begin{aligned}
& \dot{x}_{1}=-a(t) x_{1}-b(t) x_{2} \\
& \dot{x}_{2}=b(t) x_{1}
\end{aligned}
$$

where $a, b: \mathbb{R}_{\geq 0} \rightarrow \mathbb{R}$ are piece-wise continuous functions defined as follows. Let $b(t):=1$ for all $t \geq 0$ and, for an arbitrary but fixed constant $T>0$ and for each $n \geq 1$, let us introduce the function $a_{n}: \mathbb{R}_{>0} \rightarrow\{0,1\}$ defined as

$$
a_{n}(t):= \begin{cases}1 & \forall t \in\left[\frac{2 i}{n} T, \frac{2 i+1}{n} T\right] \quad i \in\left[0, \frac{n}{2}-1\right] \\ 0 & \forall t \in\left[\frac{2 i+1}{n} T, \frac{2 i+2}{n} T\right] .\end{cases}
$$

That is, $a_{n}(t)$ is a square train pulse with a $50 \%$ duty cycle; it is a periodic function of period $2 T / n$.

A simple computation shows that, for any $n \geq 1$,

$$
\left|\int_{t}^{t+2 T} b(s) a_{n}(s) d s\right|=\int_{t}^{t+2 T}\left|b(s) a_{n}(s)\right| d s=T
$$

for all $t \geq 0$. In particular, $a_{n}$ is persistently exciting. Moreover, the same condition holds for the function

$$
a(t):=a_{n}(t) \quad \forall t \in[(n-1) T, n T], \quad n \geq 0 .
$$

Furthermore, the condition (10) with $\Omega_{i}=a_{n}(t)$ holds for any fixed $n$, but it fails as $n$ increases since the lengths of the intervals over which $a(t)=1$ diminish arithmetically. In other words, $a(t)$ is a train of square pulses of $50 \%$ duty cycle but of linearly increasing frequency.

More precisely, there exist strictly positive constants $T, T_{0}, \mu$, and, for any $t \geq 0$, there exists $t^{*} \in[t, t+T]$, such that

$$
\left|\int_{t^{*}}^{t^{*}+T_{0}} a(s) b(s) d s\right| \geq \mu .
$$

but there does not exist $\underline{a}>0$ such that

$$
a(s) \geq \underline{a} \quad \forall s \in\left[t^{*}, t^{*}+T_{0}\right] .
$$

As a consequence, we are able to show that for this choice of $a$ and $b$ the origin of (15) is not uniformly exponentially stable. 
We have thus established that (20) is a tight condition to ensure uniformity of the convergence; indeed, without this condition the origin may still be attractive but not uniformly exponentially, as we illustrate in the coming section. See also [24]. Furthermore, if $a(t) \geq 0$ and $a(t) b(t)=0$ the trajectories for some initial conditions will not converge, see [25]. This shows that the conditions for stability imposed in Lemma 1 are weak and, consequently, so are those imposed in Assumptions 1 and 2 in the following main result for synchronization. We further illustrate these facts with some concrete simulation tests.

Theorem 1 (Main statement): Consider the linear timevarying closed-loop system (8). Under Assumption 1 and Assumption 2 with $\Omega$ therein replaced by $W_{t}$, the network (1) is uniformly exponentially synchronized, that is, for all $i, j \leq n, x_{i}(t) \rightarrow x_{j}(t)$ and $z_{i}(t) \rightarrow z_{j}(t)$ with a uniform (in $t$ ) exponential convergence rate.

Stketch of proof. After [22], since the graph $\mathcal{G}$ is bidirectional, one can explicitly compute a constant orthogonal matrix $T_{\mathcal{G}} \in \mathbb{R}^{(n-1) \times(n)}$ of rank $n-1$, such that, using the following transformation

$$
\left[\begin{array}{l}
\tilde{x} \\
\tilde{z}
\end{array}\right]:=\left[\begin{array}{cc}
T_{\mathcal{G}} & 0 \\
0 & T_{\mathcal{G}}
\end{array}\right]\left[\begin{array}{l}
x \\
z
\end{array}\right],
$$

the system (8) is equivalent to

$$
\left[\begin{array}{c}
\dot{\tilde{x}} \\
\dot{\tilde{z}}
\end{array}\right]=\left[\begin{array}{cc}
-\lambda_{\mathcal{G}} V_{\mathcal{G}}^{\top} W(t) V_{\mathcal{G}} & -b(t) I_{n-1} \\
b(t) I_{n-1} & 0
\end{array}\right]\left[\begin{array}{c}
\tilde{x} \\
\tilde{z}
\end{array}\right]
$$

where $\lambda_{\mathcal{G}} \in \mathbb{R}^{(n-1) \times(n-1)}$ is diagonal positive definite, $V_{\mathcal{G}} \in$ $\mathbb{R}^{(n-1) \times m}$ is an orthogonal matrix, and both $\lambda_{\mathcal{G}}$ and $V_{\mathcal{G}}$ are determined explicitly. Then, after decomposing the matrix $W(t)$ as $W(t)=$ blockdiag $\left\{W_{t}(t), W_{c}(t)\right\}$, where $W_{t}(t) \in$ $\mathbb{R}^{(n-1) \times(n-1)}$ corresponds to the edge weights of a spanning tree whose entries $W_{t i}(t)$ satisfy Assumption 2, we obtain

$$
\begin{aligned}
V_{\mathcal{G}}^{\top} W(t) V_{\mathcal{G}} & =\left[\begin{array}{ll}
V_{\mathcal{G}}^{1^{\top}} & V_{\mathcal{G}}^{2^{\top}}
\end{array}\right]\left[\begin{array}{cc}
W_{t}(t) & 0 \\
0 & W_{c}(t)
\end{array}\right]\left[\begin{array}{c}
V_{\mathcal{G}}^{1} \\
V_{\mathcal{G}}^{2}
\end{array}\right] \\
& =V_{\mathcal{G}}^{1^{\top}} W_{t}(t) V_{\mathcal{G}}^{1}+V_{\mathcal{G}}^{2^{\top}} W_{c}(t) V_{\mathcal{G}}^{2} \\
& \geq V_{\mathcal{G}}^{1^{\top}} W_{t}(t) V_{\mathcal{G}}^{1} .
\end{aligned}
$$

Furthermore, using the change of variable

$$
Z:=\left[\begin{array}{cc}
\lambda_{\mathcal{G}}^{-\frac{1}{2}} & 0 \\
0 & \lambda_{\mathcal{G}}^{-\frac{1}{2}}
\end{array}\right]\left[\begin{array}{l}
\tilde{x} \\
\tilde{z}
\end{array}\right],
$$

we obtain in the new coordinates

$$
\dot{Z}=-\left[\begin{array}{cc}
\lambda_{\mathcal{G}}^{\frac{1}{2}} V_{\mathcal{G}}^{\top} W(t) V_{\mathcal{G}} \lambda_{\mathcal{G}}^{\frac{1}{2}} & -b(t) I_{n-1} \\
b(t) I_{n-1} & 0
\end{array}\right] Z .
$$

The interest of this system is that it is of reduced dimension and Lyapunov's first method can be used to ascertain the stability of the origin. More precisely, uniform exponential synchronization of (8) follows if for the system (25) the origin is uniformly globally exponentially stable. To establish the latter, we observe that the system (25) is of the form (12); hence, Lemma 1 applies to system (25) with $A(t):=$
$\lambda_{\mathcal{G}}^{\frac{1}{2}} V_{\mathcal{G}}^{\top} W(t) V_{\mathcal{G}} \lambda_{\mathcal{G}}^{\frac{1}{2}}, V:=V_{\mathcal{G}}^{1} \lambda_{\mathcal{G}}^{\frac{1}{2}}$ and $\Omega:=W_{t}$; note that the condition (14) holds under (23), so

$$
\lambda_{\mathcal{G}}^{\frac{1}{2}} V_{\mathcal{G}}^{\top} W(t) V_{\mathcal{G}} \lambda_{\mathcal{G}}^{\frac{1}{2}}-\left(\lambda_{\mathcal{G}}^{\frac{1}{2}} V_{\mathcal{G}}^{1^{\top}}\right) W_{t}(t)\left(V_{\mathcal{G}}^{1} \lambda_{\mathcal{G}}^{\frac{1}{2}}\right) \geq 0,
$$

while condition (H1) holds under Assumption 1.

Remark 1: Consider the system (12) under the conditions in Lemma 1. After applying the change of coordinate

$$
z:=\mathrm{e}^{-R_{o} q(t)}\left[\begin{array}{l}
x_{1} \\
x_{2}
\end{array}\right]
$$

where $R_{o}:=\left[\begin{array}{cc}0 & -I_{n} \\ I_{n} & 0\end{array}\right]$ and $q(t):=\int_{0}^{t} b(s) d s$, the system's dynamics becomes

$$
\dot{z}=e^{-R_{o} q(t)}\left[\begin{array}{cc}
-A(t) & 0 \\
0 & 0
\end{array}\right] e^{R_{o} q(t)} z,
$$

or, equivalently,

$$
\begin{aligned}
\dot{z} & =-g(t) g(t)^{\top} z \\
g(t) g(t)^{\top} & :=\left[\begin{array}{cc}
A(t) \sin ^{2}(t) & -A(t) \sin (t) \cos (t) \\
-A(t) \sin (t) \cos (t) & A(t) \cos ^{2}(t)
\end{array}\right] .
\end{aligned}
$$

The origin for (28) is uniformly exponentially stable if and only if $g(t) g(t)^{\top}$ is persistently exciting, that is, if there exists $\mu_{o}>0$ and $T_{o}>0$ such that

$$
\int_{t}^{t+T_{o}} g(s) g(s)^{\top} d s \geq \mu_{o} I_{N} \quad \forall t \geq 0 .
$$

Furthermore, we are able to deduce explicit estimates of the excitation parameters $\left(T_{o}, \mu_{o}\right)$ for the resulting matrix $g(t) g(t)^{\top}$ such that (29) holds. Hence, after [16], one can explicitly estimate the convergence rate for the system (28) using the following strict Lyapunov function:

$$
\begin{aligned}
V(t, z) & :=\frac{1}{2} z^{\top}[\alpha+Q(t)] z, \quad \alpha:=1+\frac{2 T_{o}^{3}\left|g(t) g(t)^{\top}\right|_{\infty}^{3}}{\mu_{o}}, \\
Q(t) & :=T_{o}\left|g(t) g(t)^{\top}\right|_{\infty}+\frac{2}{T_{o}} \int_{t}^{t+T_{o}} \int_{m}^{t} g(s) g(s)^{\top} d s d m .
\end{aligned}
$$

Indeed, it can be verified that, along the trajectories of (28),

$$
\dot{V}(t, z) \leq-\frac{\mu_{o}}{2 T_{o}}|z|^{2} .
$$

\section{Simulation RESUlts}

In order to illustrate our results we have performed simulation tests under Simulink ${ }^{T M}$ of Matlab ${ }^{T M}$.

In a first simulations test we consider the network (1) composed by only two oscillators interconnected via the control law (2). The weight of the links are set to $a_{12}(t)=$ $a_{21}(t)=: w_{1}(t)$, which is designed to be persistently exciting - see Figure 1. In addition, as shown in the latter figure, the product $w_{1}(t) b(t)$ is also persistently exciting and both (19) and (20) hold.

As expected, after Theorem 1, the oscillators trajectories synchronize — see Figure 2; the phase portraits are shown in Figure 3. 

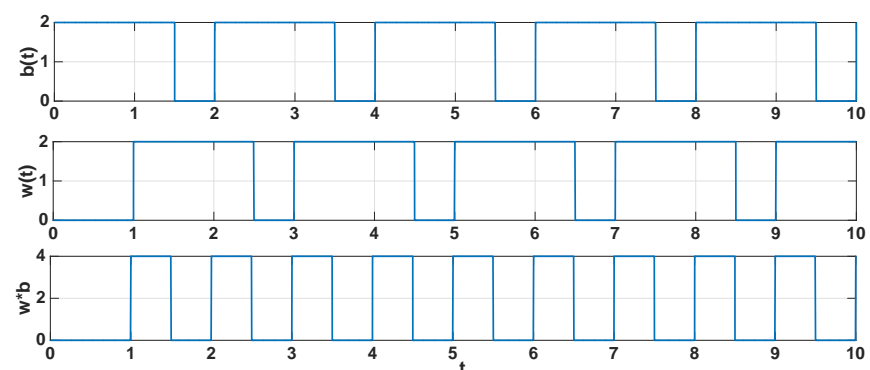

Fig. 1. Weight of the communication link and frequency of the oscillators
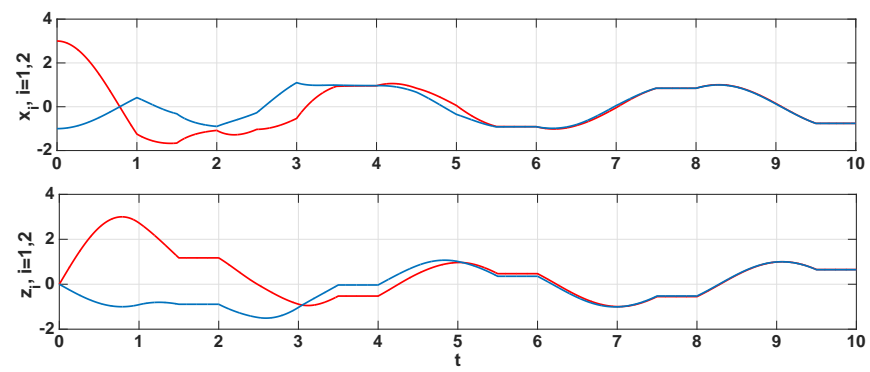

Fig. 2. Synchronization of the network trajectories in closed loop

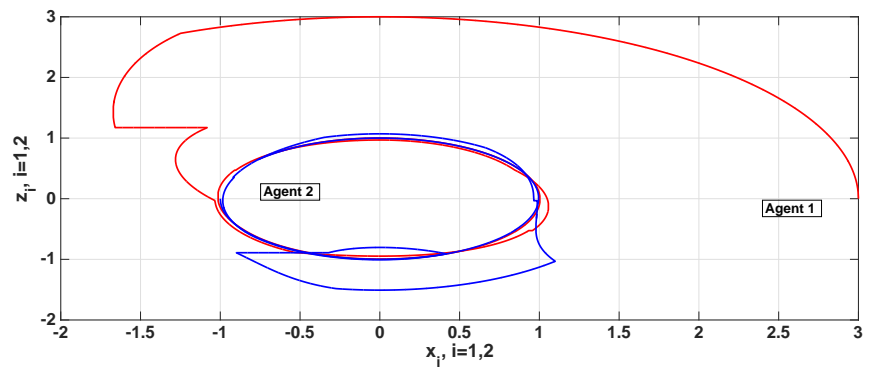

Fig. 3. Phase portrait of the network components in closed loop

Next, in order to illustrate the importance of condition (20) we simulate the trajectories of the system (15) and we compare the convergence rates of the error coordinates $e=$ $\left(x_{1}-x_{2}, z_{1}-z_{2}\right)$, when the weight function $w_{1}$ is constructed according to (18); see the illustration in Figure 4. Note from the latter that $w_{1}(t)$ is bounded with with bounded derivative almost everywhere and it satisfies (19), but it does not verify the condition (20) since the length of the intervals over which $w_{1}=1$ shrinks as $t \rightarrow \infty$. As a result, the convergence of the error coordinates $e=\left(x_{1}-x_{2}, z_{1}-z_{2}\right)$ to the origin depends on the choice of the initial time and becomes slower when the initial time increase. This fact, which may be appreciated in Figure 5 confirms the loss of uniformity in the convergence rate, as established above.

Finally, in a third simulations test, we consider the network (1) composed of four planar time-varying oscillators with time varying interconnections, as illustrated in Figure 6.

The consensus control law (2) is used and the sequence of edge weights $w_{1}(t), w_{2}(t), w_{3}(t)$ forming a spanning tree are illustrated in Figure 7 and shown in Figure 8, so the conditions of Theorem 1 hold.
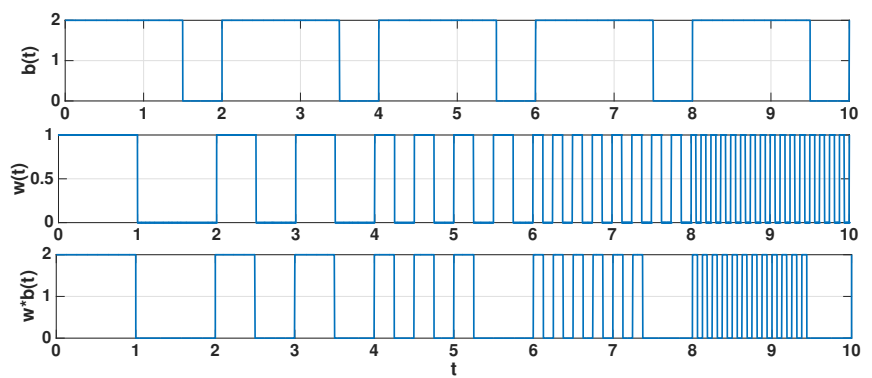

Fig. 4. The pathological weight sequence constructed in (5) and oscillators' frequency

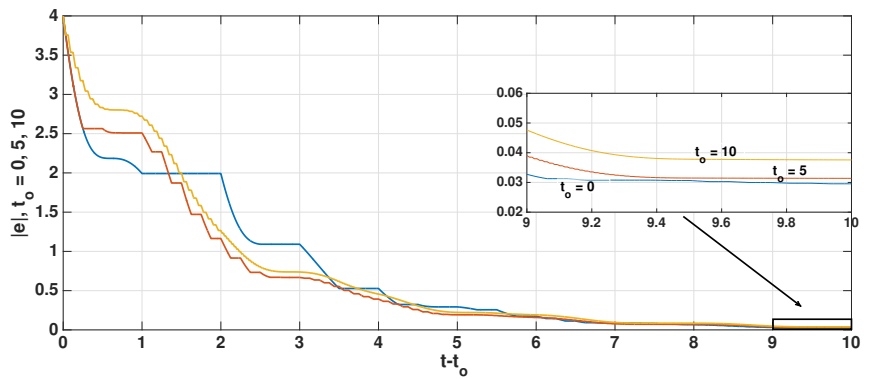

Fig. 5. Convergence rate comparison for different values of $t_{o}$ under in the the weight sequence (5)

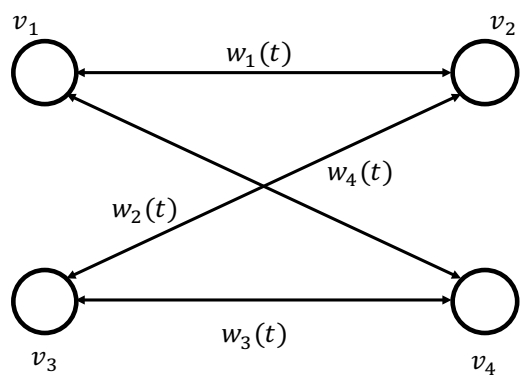

Fig. 6. Topology of interconnection for the network of four oscillators
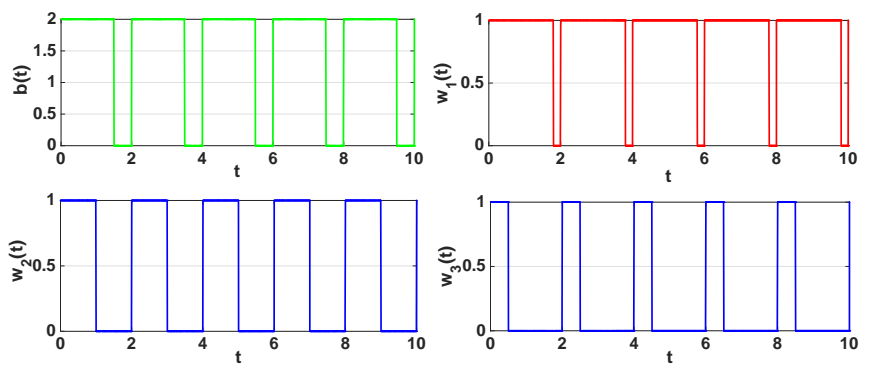

Fig. 7. Weights of the edges forming a spanning tree and frequency of the oscillators

The full synchronization among the components of the network in steady state may be appreciated from Figure 9. This can be seen also from the phase portraits in Figure 10.

\section{CONCLUSION}

We presented a decentralized controller that guarantees uniform exponential synchronization for networks of planar harmonic oscillators with time-varying frequency, intercon- 

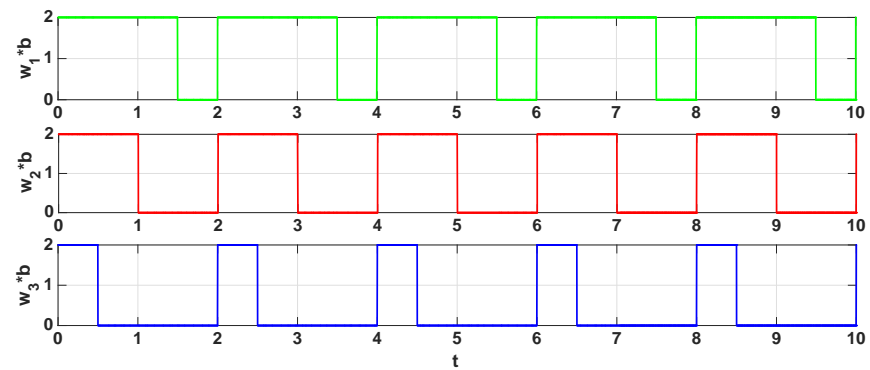

Fig. 8. Product between weights of the edges forming a spanning tree and the oscillators' frequency
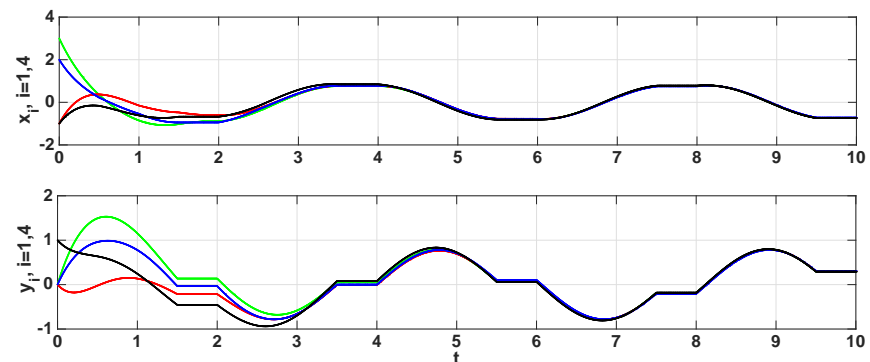

Fig. 9. Synchronization of the network trajectories in closed loop

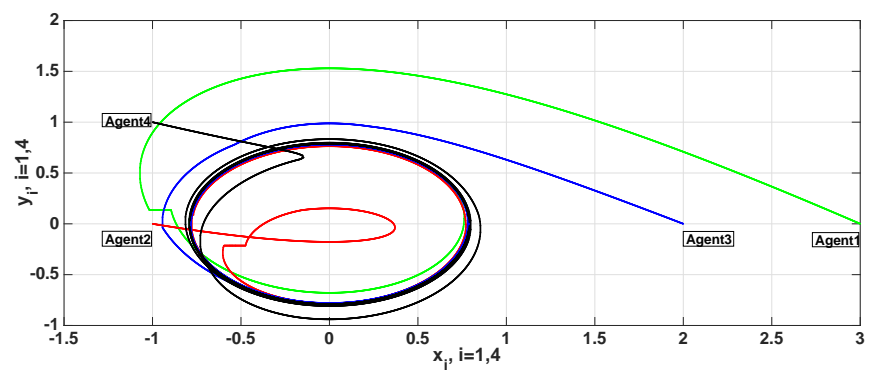

Fig. 10. Phase portrait of the network components in closed loop

nected over a bidirectional graph with time-varying topology. Our conditions are stated in terms of persistency of the connectivity and we show that this is intrinsically related to the oscillators frequency.

\section{REFERENCES}

[1] W. Ren, R. W. Beard, and E. M. Atkins, "A survey of consensus problems in multi-agent coordination," in Proc. of the American Control Conference, pp. 1859-1864, IEEE, 2005.

[2] W. Ren and R. Beard, "Decentralized scheme for spacecraft formation flying via the virtual structure approach," Journal of Guidance, Control, and Dynamics, vol. 27, no. 1, pp. 73-82, 2004.

[3] K. D. Do and J. Pan, "Nonlinear formation control of unicycle-type mobile robots," J. Rob. and Aut. Syst., vol. 55, no. 3, pp. 191-204, 2007.

[4] D. J. W. Belleter and K. Y. Pettersen, "Leader-follower synchronisation for a class of underactuated systems," in Nonlinear Systems, pp. 157-179, Springer International Publishing, 2017.
[5] G. L. Mariottini, F. Morbidi, D. Prattichizzo, N. V. Valk, N. Michael, G. Pappas, and K. Daniilidis, "Vision-based localization for leaderfollower formation control," IEEE Trans. on Robotics, vol. 25, no. 6, pp. 1431-1438, 2009.

[6] A. Jadbabaie, J. Lin, and A. S. Morse, "Coordination of groups of mobile autonomous agents using nearest neighbor rules," IEEE Trans. on Automatic Control, vol. 48, no. 6, pp. 988-1001, 2003.

[7] R. R. Olfati-Saber and R. M. Murray, "Consensus problems in networks of agents with switching topology and time-delays," IEEE Trans. on Automatic Control, vol. 49, no. 9, pp. 1520-1533, 2004.

[8] L. Moreau, "Stability of continuous-time distributed consensus algorithms," in Proc. of the 43rd. IEEE Conf. on Decision and Control, vol. 4, pp. 3998-4003, 2004.

[9] M. Mesbahi and M. Egerstedt, Graph theoretic methods in multiagent networks. Princeton University Press, 2010.

[10] N. Alvarez-Jarquin, A. Loría, and J. L. Avila, "Consensus under switching spanning-tree topologies and persistently exciting interconnections," in Proc. IEEE American Control Conference, (Milwaukee, WI, USA), 2018.

[11] W. Ren and R. W. Beard, "Consensus seeking in multiagent systems under dynamically changing interaction topologies," IEEE Trans. on Automatic Control, vol. 50, no. 5, pp. 655-661, 2005.

[12] W. Ren, "Synchronization of coupled harmonic oscillators with local interaction," Automatica, vol. 44, no. 12, pp. 3195-3200, 2008.

[13] J. Zhou, H. Zhang, L. Xiang, and Q. Wu, "Synchronization of coupled harmonic oscillators with local instantaneous interaction," Automatica, vol. 48, no. 8, pp. 1715-1721, 2012.

[14] L. Moreau, "Stability of multiagent systems with time-dependent communication links," IEEE Trans. on Automatic Control, vol. 50, pp. 169-182, Feb 2005.

[15] S. Martin and A. Girard, "Continuous-time consensus under persistent connectivity and slow divergence of reciprocal interaction weights," SIAM Journal on Control and Optimization, 2013.

[16] N. R. Chowdhury, S. Sukumar, M. Maghenem, and A. Loria, "On the estimation of algebraic connectivity in graphs with persistently exciting interconnections," Int. J. of Contr., vol. 91, no. 1, pp. 132144, 2018.

[17] B. D. O. Anderson, G. Shi, and J. Trumpf, "Convergence and state reconstruction of time-varying multi-agent systems from complete observability theory," IEEE Trans. on Automatic Control, vol. 62, no. 5, pp. 2519-2523, 2017.

[18] L. Scardovi and R. Sepulchre, "Brief paper: Synchronization in networks of identical linear systems," Automatica (Journal of IFAC), vol. 45, no. 11, pp. 2557-2562, 2009.

[19] K. S. Narendra and A. M. Annaswamy, Stable adaptive systems. New Jersey: Prentice-Hall, Inc., 1989.

[20] A. P. Morgan and K. S. Narendra, "On the stability of nonautonomous differential equations $\dot{x}=[A+B(t)] x$ with skew-symmetric matrix $B(t)$," SIAM J. on Contr. and Opt., vol. 15, no. 1, pp. 163-176, 1977.

[21] A. P. Morgan and K. S. Narendra, "On the uniform asymptotic stability of certain linear nonautonomous differential equations," SIAM J. on Contr. and Opt., vol. 15, no. 1, pp. 5-24, 1977.

[22] S. S. N. R. Chowdhury and N. Balachandran, "Persistence based convergence rate analysis of consensus protocols for dynamic graph networks," European Journal of Control, vol. 29, pp. 33-43, 2016.

[23] N. Barabanov and R. Ortega, "On global asymptotic stability of $\dot{x}=$ $-\phi(t) \phi(t)^{\top} x$ with $\phi$ not persistently exciting," Systems and Control Letters, no. 109, pp. 24-29, 2017.

[24] N. B. R. Ortega and J. Wang, "On global asymptotic stability of $\dot{x}=-\phi(t) \phi(t) x$ with $\phi(t)$ bounded and not persistently exciting," Submitted to System and control letters, 2017.

[25] M. Maghenem and A. Loría, "Strict Lyapunov functions for timevarying systems with persistency of excitation," Automatica, vol. 78 , pp. 274-279, 2017. 